\title{
On the energy detection performance based Welch's DCT algorithm in cognitive radio systems
}

\begin{abstract}
Currently, modern devices and applications for communications need huge frequency bands since it has limited resources. To address this issue, the cognitive radio is considered a promised solution where its main function is exploiting the idle spectrum using one of spectrum sensing (SS) techniques. A energy detection (ED) for SS technique that handles low signal-to-noise ratio (SNR) signals is proposed in this paper. It is derived using the discrete cosine transform (DCT) according to Welch's periodogram to estimate the power spectral density (PSD). The proposed technique is applied to sense the digital video broadcast (DVB) via AWGN channel with various SNR values and various user terminals. The results of the proposed technique proved that its performance is better than the conventional Welch's algorithm for both cases of low SNR and multi user terminals.
\end{abstract}

Keyword: Spectrum sensing technique; Cooperative spectrum sensing; Energy detection; DCT; PSD; Welch's algorithm; Low SNR 